\title{
Errata
}

\section{Evidence for the Poisson Distribution for Quasi-Energies in the Quantum Kicked-Rotator Model $^{1}$}

\section{A. Pellegrinotti}

Because of postal delay corrections in the galley proof were not incorporated in this paper. Following is a list of corrections:

p. 1327: all "r.w." should read "r.v."

p. 1328: "r.w." should read "r.v."

p. 1330: the formula inside the integral should read

$$
\begin{aligned}
& \int_{0}^{1} \int_{0}^{1} d x d y \exp \left[2 \pi i\left(m_{1}+m_{3}+\cdots+m_{2(n-1)-1}\right) x\right. \\
& \left.+2 \pi i\left(m_{2}+m_{4}+\cdots+m_{2(n-1)}\right) y\right]
\end{aligned}
$$

one summation index should read

$$
m_{2}\left(k_{2}-k_{1}\right)-m_{1}=0
$$

in the first r.h.s. member of the last equality there is a missing set of parentheses

$$
\cdots \exp (-2 \pi i \varepsilon m)-1 \ldots
$$

p. 1332: the second addend in the r.h.s. of formula (3.4) should read

$$
4 c\left(\varepsilon^{3} \sum_{\substack{k_{1}, k_{2}, k_{3} \\ 0 \leqslant k_{i} \leqslant c / \varepsilon}} \sum_{\substack{m_{1}, m_{2}, m_{3} \\ m_{i} \neq 0}} h\left(\varepsilon m_{1}\right) h\left(\varepsilon m_{2}\right) h\left(\varepsilon m_{3}\right)\right)
$$

\footnotetext{
${ }^{1}$ This paper appeared in J. Stat. Phys. 53:1327 (1988).
} 
p. 1333: in formula (3.7), the coefficient of $k_{1}^{2}$ is $m_{1}\left(m_{1}+m_{3}\right)$; in the formula for $\Delta$ the term of place 3,3 is $a_{1}^{2}-m_{3} a_{2}$

p. 1335: in the r.h.s. of the inequality, $\varepsilon^{2}$ should read $\varepsilon^{3}$

p. 1336: The integral in the last formula is

$$
\int_{R^{3}} \frac{\cos (x+y+z)-\cos (x+y)-\cos (x+z)-\cos (y+z)+\cos x+\cos y+\cos z-1}{x y z(x+y+z)} d x d y d z
$$

\section{The Reunions of Three Dissimilar Vicious Walkers ${ }^{2}$}

\section{E. Fisher and M. P. Gelfand}

On page 185, equation (3.4) should read, correcting subscripts in the two factors in parentheses,

$$
C_{3}\left(b_{1} \rightarrow 0, b_{2}=b_{3}\right)=x_{12} x_{13} x_{12,0} x_{13,0}\left(x_{13}^{2}-x_{12}^{2}\right)\left(x_{13,0}^{2}-x_{12,0}^{2}\right) / 3 \pi b^{8}
$$

In the first line of the following paragraph, the limit $b_{3} \rightarrow 0$ should be replaced by $b_{2} \rightarrow 0$.

${ }^{2}$ This paper appeared in J. Stat. Phys. 53:175 (1988). 